\title{
An Energy Balanced Clustering Algorithm Based on LEACH Protocol
}

\author{
Qian Liao, Hao Zhu
}

School of Information Engineering, Zhengzhou Univesity, Zhengzhou, China

E-mail:liaoqian2006521@163.com

Keywords: WSN; LEACH; cluster-head selection; energy balanced; network lifetime

\begin{abstract}
The primary objectives of the wireless sensor network routing protocol design are balancing network energy consumption and extending the entire network lifetime. This paper analyses the effectiveness of LEACH protocol in cluster-head selection, and proposes an improved clustering algorithm. This new algorithm takes node's residual energy and location information into account, optimizes the selection method of the threshold for electing cluster-head, improves optimal cluster-head selection strategy that is normal nodes select the optimal cluster-head based on the cost function. Simulation results show that the improved protocol is better than LEACH in balancing node energy consumption, improving the efficiency of data transmission and prolonging the network life.
\end{abstract}

\section{Introduction}

Wireless sensor network (WSN) [1] is a self-organized network composed by a large number of micro sensors that are randomly deployed in monitoring regional through wireless communication. With its wide application in military reconnaissance, medical aid, logistics management, environmental monitoring, agriculture and other commercial areas, WSN has become the furthermost technology in the field of communication and computer research [2]. Sensor nodes rely on battery power supply, their communication capability and energy storage capacity are very limited, so how to utilize the energy of nodes efficiently, balance the network energy consumption and extend the network lifetime has become a primary design objective for wireless sensor network.

Among the current researches, the clustering routing technology is the most widely influential. Low-Energy Adaptive Clustering Hierarchy (LEACH) is a classical clustering routing in wireless sensor networks [3]. However the cluster-head selection in LEACH protocol is lack of balancing the whole network energy consumption, with the result that low energy nodes run out of energy prematurely and decline the network life. This paper analyses the effectiveness of LEACH protocol in cluster-head selection, and proposes an improved energy balanced clustering algorithm.

\section{LEACH Protocol}

\section{Protocol Description}

LEACH is the earliest proposed single-hop clustering routing protocol in WSN, it can save network energy greatly compared with the non-cluster routing algorithm. Many other clustering algorithm are proposed based on LEACH, such as TEEN (Threshold Sensitive Energy Efficient Sensor Network Protocol)[4], PEGASIS(Power Efficient Gathering in Sensor Information Systems)[5] , HEED(Hybrid Energy-Efficient Distributed Clustering)[6] and so on. In LEACH protocol, all clusters are self-organized, each cluster contains a cluster-head and several non-cluster head nodes, cluster-head node consumes more energy than non-cluster head nodes. With the purpose of balancing network energy consumption and prolonging the network life cycle, it selects cluster head randomly and each node has an equal chance to be cluster-head[7]. The cluster structure update constantly in operation, and one updating process is called a round. The cycle of each round contains two stages: set-up phase and steady-state phase, set-up phase is the establishment phase of the cluster, steady-state phase is the stable data transfer phase.

Set-up phase;each node generates a random number between 0 to 1 , and compares this number with the threshold value $T(n)$. If the number is less than $T(n)$, the node is selected as a cluster-head, 
the threshold $T(n)$ is set as follow;

$$
T(n)= \begin{cases}\frac{p}{1-p^{*}\left(r \bmod \frac{1}{p}\right)} & \text { if } n \in G \\ 0 & \text { if } \mathrm{n} \notin \mathrm{G}\end{cases}
$$

Where $n$ refers the node identification in the current sensor network; $p$ is the percentage of cluster-heads; $r$ is the current round number; $G$ is the set of nodes that have not been elected as cluster-head in the last $1 / p$ rounds. Once the cluster-head is determined, the cluster-head sends a broadcast message to the network, announced itself as the cluster-head, each normal node decides which cluster to join in according to the signal strength of the received message, sends a request message to the corresponding cluster-head. The cluster-head receives all the messages sent by the nodes that would like to join in the cluster, confirms them as members of the cluster, then joins them in the routing table and allocates TDMA table of slots for the cluster members telling each member when it can transmit data.

Steady-state phase: members of the cluster send data to the cluster-head in the way of single-hop during the allocated slot according to the TDMA table, the cluster-head receives all the data from each node in the cluster, fuses all the data into a single signal, after that the fusion signal is transmitted to the base station by cluster-head. Data transmission lasts a certain time, and then the entire network comes into the next round.

\section{Radio Signal Propagation Model}

This paper deals with the first order radio frequency energy consumption model to describe energy feather of the communication channel [8]. The first order radio model can be divided into free-space model and multi-path fading model according to the distance between the sending node and receiving node. The protocol assumes that the communication channel is symmetrical, the energy consumption of $l$ bits message between two nodes with a distance of $d$ can be shown as equations (2) and (3).

$$
\begin{aligned}
& E_{T x}(l, d)=\left\{\begin{array}{ll}
E_{\text {elec }} * l+\varepsilon_{f s} * l * d^{2} & d \leq d_{0} \\
E_{\text {elec }} * l+\varepsilon_{\text {mp }} * l^{*} d^{4} & d>d_{0}
\end{array},\right. \\
& E_{R x}(l)=E_{\text {elec }} * l .
\end{aligned}
$$

Where $E_{T x}(l, d)$ is the energy consumption in transmitting $l$ bits data to a node with a distance of $d, E_{R x}(l)$ is the energy consumption in receiving $l$ bits data. $E_{\text {elec }}$ equals the per bit energy consumption for transmitter and receiver circuit . $\varepsilon_{m p}$ and $\varepsilon_{f s}$ are the amplifier parameters of transmission corresponding to the multi-path fading model and the free-space model respectively. $d_{0}$ is the threshold distance between multi-path fading model and the free-space model, $d_{0}=\sqrt{\frac{\varepsilon_{f s}}{\varepsilon_{m p}}}$. If $d \leq d_{0}$, the channel approximates free-space model, the energy dissipation in transmitter amplifier is in direct ratio to $d^{2}$. If $d>d_{0}$, the multi-path fading model will be employed and the energy dissipation is in direct ratio to $d^{4}$.

\section{The advantages and disadvantages of LEACH protocol}

LEACH protocol has a relatively good function in energy consumption through dynamic clustering, keeps the data transmission in cluster which reduces the energy consumption by communicating directly between nodes and the base station, but there are still a lot of inadequacies.

The LEACH protocol uses the mechanism of cluster-head rotation, elects cluster-head randomly, after several rounds of data transmission, the residual energy of the nodes will have a great difference, cluster-head or the nodes which are far from the base station will consume more energy 
in transmitting data of the same length relatively, if they are selected as cluster-heads after that, they will run out of energy and become invalid. Once the number of invalid nodes increases, it'll have a great influence in the network performance and shorten the life of the network.

Cluster member nodes select the optimal cluster-head based on the received signal intensity to join in, do not consider the distance from the node itself to the base station, either the distance between cluster-head and the base station. So normal node may chose the cluster-head that is far from base station as its optimal cluster-head, this not only is the heavy burden to the cluster-head but also increases the extra energy consumption, which is not beneficial to balance network energy consumption.

\section{Improvement of the LEACH Protocol}

This paper considers node energy and position information to improve the LEACH algorithm, proposes an energy balanced clustering algorithm named L-LEACH.

\section{Cluster-head Threshold Optimization}

There are many improved methods of the cluster-head election based on residual energy, the threshold in document [2] is shown as the following equation:

$$
T(n)=\frac{p}{1-p^{*}\left(r \bmod \frac{1}{p}\right)} * \frac{E_{\text {cur }}}{E_{0}},
$$

$E_{\text {cur }}$ is the current energy of node, $E_{0}$ is the node initial energy. This improvement takes the current energy into consideration, and increases the probability of the high-energy nodes to become cluster-head, but there is also a significant problem. When the remaining energy is very low, threshold $T(n)$ becomes very small, the probability of the node random number being smaller than the threshold becomes small, the cluster-head nodes in the network will be too little, the selected cluster-head consumes too much energy and thus affects the network life because of the untimely death. What's more, the above method doesn't consider the influence of the distance between nodes and the base station in electing cluster-head. The improved threshold in this paper is:

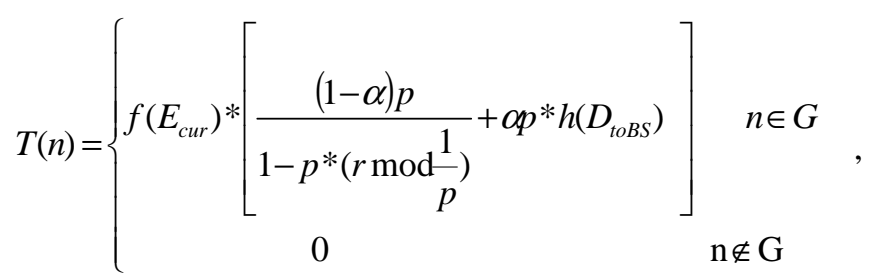

$f\left(E_{\text {cur }}\right)$ is the function relevant to the current residual energy of the node, it reflects the influence of node energy to the elected cluster-head probability. $h\left(D_{\text {toBs }}\right)$ is the $D(n)$ 's impact on cluster-head election, increasing the probability of the near-by base station node becoming cluster-head. $\alpha$ is the influencing factor. $f\left(E_{c u r}\right)$ and $h\left(D_{t o B S}\right)$ are presented in equations (6)and(7), in which $E_{\text {ave }}$ is the average remaining energy of all nodes in the current round, $D_{\max }$ and $D_{\min }$ present the maximum and minimum of the distance between all nodes and the base station respectively.

$$
\begin{aligned}
& f\left(E_{\text {cur }}\right)=\frac{E_{\text {cur }}}{E_{\text {ave }}}, \\
& h\left(D_{\text {toBS }}\right)=\frac{D_{\text {max }}-D(n)}{D_{\text {max }}-D_{\text {min }}} .
\end{aligned}
$$

Improvement the strategy of non-cluster head node selecting the optimal cluster-head 
In LEACH protocol, the normal nodes in the cluster select the optimal cluster-head and join in this cluster, then communicate with the base station for data transmission through the cluster-head .The improved algorithm considers the distance between normal node and the base station, compares the distances from the node to the cluster-head and the base station. If the node is closest to the base station, no optimal cluster-head will be selected and it will directly send controlling packages to the base station and then transmit data packages. Otherwise, the cluster-heads whose distance to the node is smaller than the distance from the node to the base station are regarded as candidate cluster-heads. Then the non-cluster head node chooses the optimal one among the candidate cluster-heads according to the cost function. If the cost function value is the least, it will be chosen as the optimal cluster-head. The cost function of the normal node $p_{i}$ joins in the cluster with the cluster-head $c_{j}$ can be shown as:

$$
\operatorname{Cost}(i, j)=\frac{d_{i j}}{d_{\max }} * \frac{E_{c u r}(i)}{E_{c u r}(j)} * \frac{D(j)}{D_{\text {ave }}} .
$$

Where $d_{i j}$ is the distance from the node $p_{i}$ to cluster-head $c_{j}, d_{\max }$ is the maximum of the distances from $p_{i}$ to the candidate cluster-heads. $E_{c u r}(i)$ and $E_{c u r}(j)$ is the current residual energy of node $p_{i}$ and cluster-head $c_{j}, D(j)$ is the distance from cluster-head $c_{j}$ to the base station, $D_{\text {ave }}$ is the average distance between cluster-heads and the base station.

\section{Simulation and Result Analysis}

The network model employed in this paper is as follows:

(1) The sensor nodes are randomly distributed in a square region. After the normal nodes are deployed, the base stations and nodes are fixed and the cluster-heads rotate randomly.

(2) The energy of node may be heterogeneous, but the energy restriction can not be added, the energy and the storage capacity of the base station are not limited.

(3) Nodes can communicate not only with each other in the network, but also with the base station directly. Node can adjust the wireless transmission power according to the distance.

To evaluate the algorithm performance, we simulate both LEACH and L-LEACH by Matlab. 100 sensor nodes are randomly distributed in the $100 \mathrm{~m} \times 100 \mathrm{~m}$ square area, nodes energy are heterogeneous, the initial energy range is $0.5 \sim 1 \mathrm{~J} . E_{\text {elec }}=50 \mathrm{~nJ} / \mathrm{bit}, \varepsilon_{\mathrm{fs}}=10 \mathrm{pJ} / \mathrm{bit} / \mathrm{m}^{2}$, $\varepsilon_{\mathrm{fs}}=10 \mathrm{pJ} / \mathrm{bit} / \mathrm{m}^{2}$, the per bit energy consumption for data fusion is $E_{D A}=5 \mathrm{~nJ} / \mathrm{bit}$, the length of data packets is 6400 bits, the controlling packages length is 200 bits. As for L-LEACH algorithm, the influence factor $\alpha$ is set as 0.1 .

Assume the coordinates of the base station are $(50,50)$, the relation curve between the number of alive nodes and network running time is shown in figure 1 . The $x$-axis is the rounds number, it represents the network running time. The y-axis is the number of alive nodes, it represents the network lifetime.

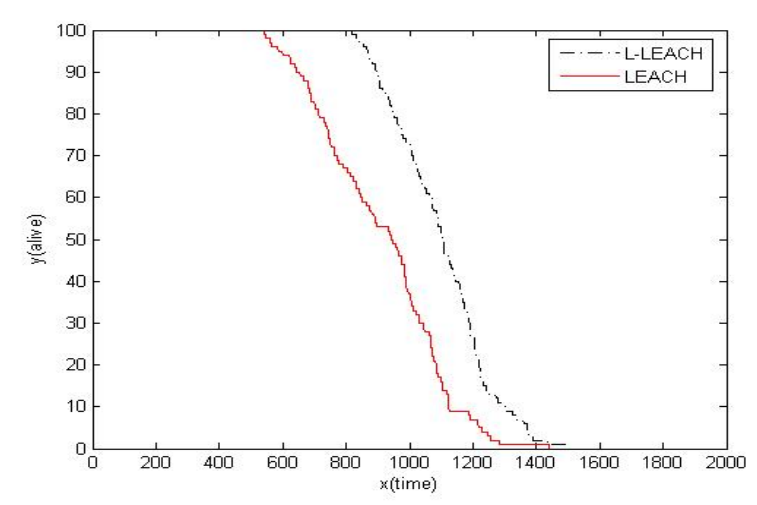


Fig. 1 The simulation of the network lifetime

As is shown in the simulation, the first node death occurs at the $542^{\text {nd }}$ round in LEACH, and the $819^{\text {th }}$ round in L-LEACH, that's to say, the first node death time is extended $51 \%$. Half of nodes die at the $943^{\text {rd }}$ round in $\mathrm{LEACH}$, and $1103^{\text {rd }}$ in $\mathrm{L}-\mathrm{LEACH}$, the improved algorithm prolongs the death time by $16 \%$. All nodes death time is $1439^{\text {th }}$ and $1497^{\text {th }}$ round respectively. All these results show that the L-LEACH algorithm can extend the network life effectively, and has better convergence.

The relation curves between the total network energy consumption, the amount of data transmission and the network running time of alive nodes are as figure 2 and 3.

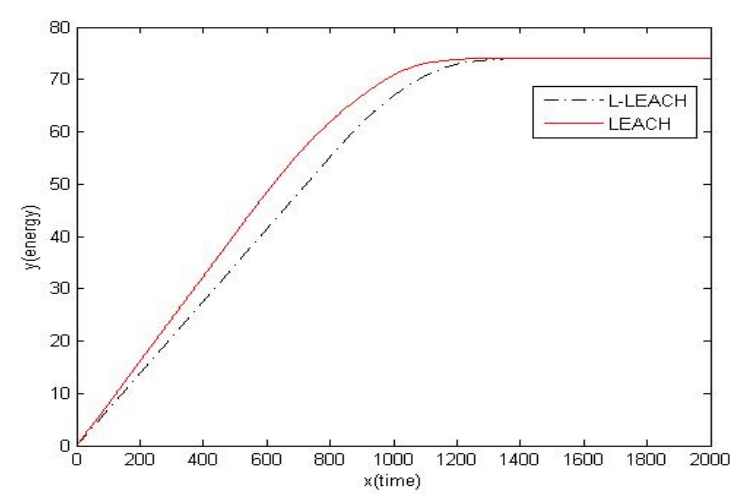

Fig. 2 The simulation of the network energy consumption

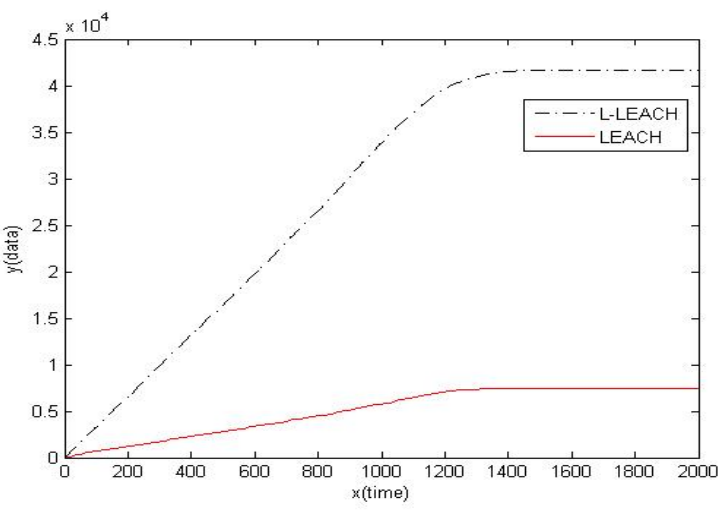

Fig. 3 The simulation of the data transmission

As can be seen, the energy consumption in L-LEACH is less than in LEACH algorithm at any time. The causes are as follows: the improved algorithm selects cluster-head based on residual energy and node coordinates information, enables each node to consume energy in a relatively balanced way, the early death of low-energy nodes is avoided, the network energy dissipation is saved and the amount of data transmission is enhanced considerably.

Consider the impact of the base station in different position on the performance of the algorithm, when the base station is located in $(50,100)$, the simulations of network life cycle and energy consumption are shown in figure 4 and 5 .

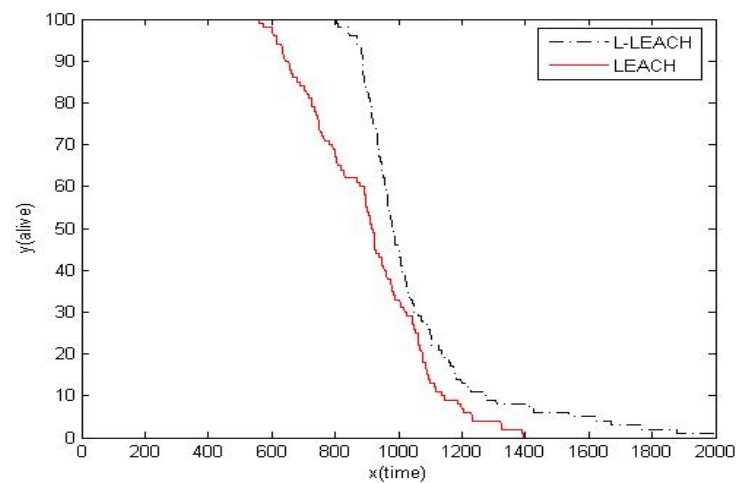

Fig. 4 The comparison of the network lifetime when base station is located in $(\mathbf{5 0 , 1 0 0 )}$

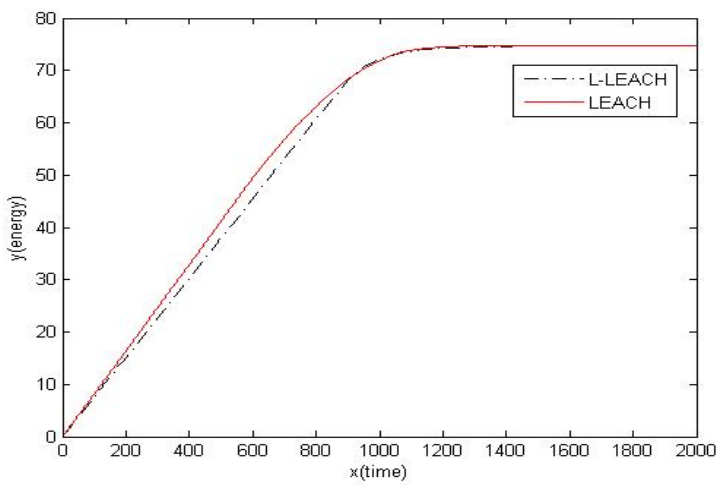

Fig. 5 The comparison of energy consumption when base station is located in $(50,100)$

When the base station is located in $(100,300)$, the comparison of two algorithms in network lifetime is shown in figure 6.

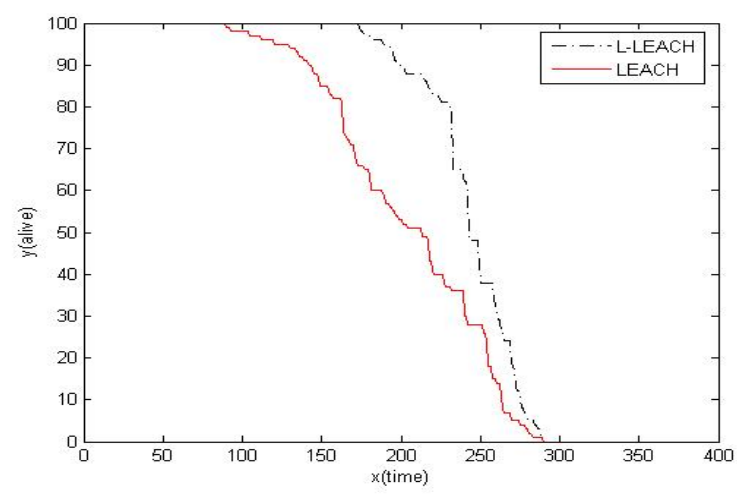


Fig. 6 The comparison of the network lifetime when base station is located in $(100,300)$

The simulation result suggests that the improved algorithm has better performance in energy balancing and network life time, it has better expansibility, so it can be used not only when the base station is near the monitoring area but also when it is far.

\section{Conclusion}

On the basis of traditional LEACH protocol, this paper proposes an energy balance algorithm optimizing cluster-head selection. This algorithm comprehensively considers the residual energy and distance factors, improves cluster-head election and the strategy of non-cluster head node selecting the optimal cluster-head. As is proved in the simulation result, the improved algorithm can effectively balance the network energy consumption, heighten system data transmission, and prolong the nodes and network life. The next focus is a combination of the improved algorithm and multi-hop routing, and paying more attention to the complexity of the algorithm and network overhead.

\section{References}

[1] Feng Shang, Mehran Abolhasan, Tadeusz Wysocki. An Energy-Efficient adaptive Clustering Algorithm for Wireless Sensor Networks. International Journal of Information Acquisition, 2009, 6(2): 117-126.

[2] Handy MJ, Hasse M, Timmermann D. Low energy adaptive clustering hierarchy with deterministic cluster-head selection [C]// Proc of the 4th IEEE Conf. on Mobile and Wireless Communications Networks. Stockholm, 2002:368-372.

[3] Heinzelman WR, Chandrakasan A, Balakrishnan H. An application-specific protocol architecture for wireless sensor networks [J]. IEEE Transaction on Wireless Communications, 2002, 1:660-670.

[4] Manjeshwar A, Grawal D.P. TEEN: A protocol for enhanced efficiency in wireless sensor networks[C].Proceeding of the 15th Parallel and Distributed Processing Symp. San francisoi, 2001: 2009-2015.

[5] Lindsey S, Raghavenda CS. PEGASIS: Power efficient gathering in sensor information systems[C]. Proceeding of the IEEE Aerospace Conf. NEW YORK, 2002: 1125-1130.

[6] Younis O, Fahmy S. HEED: A hybrid, energy-efficient, distributed clustering approach for ad-hoc sensor networks [J]. IEEE Trans. On Mobile Computing.2004, 3(4):660-669.

[7] LI-Qing GUO, YI XIE*, CHEN-HUI YANG and ZHENG-WEI JING: Improvement on LEACH by combining Adaptive Cluster Head Election and Two-hop transmission,[J]. Proceeding of the Ninth International Conference on Machine Learning and Cybernetics, Qingdao, July 2010, pp: 1678-1683.

[8] Yuhua L, Yongfeng Z, Jingju G. A New Clustering Mechanism Based on LEACH Protocol [C]// Proceedings of the International Joint Conference on Artificial Intelligence. IEEE Communications Society. 2009: 715-718. 